\title{
BMJ Open Study protocol for a multicentre implementation trial of monotherapy anticoagulation to expedite home treatment of patients diagnosed with venous thromboembolism in the emergency department
}

Jeffrey Kline (1) , ${ }^{1}$ David Adler, ${ }^{2}$ Naomi Alanis (D) , Joseph Bledsoe, ${ }^{4}$ Daniel Courtney, ${ }^{5}$ James D'Etienne, ${ }^{6}$ Deborah B Diercks, ${ }^{5}$ John Garrett, ${ }^{7}$ Alan E Jones, ${ }^{8}$ David MacKenzie, ${ }^{9}$ Troy Madsen, ${ }^{10}$ Andrew Matuskowitz, ${ }^{11}$ Bryn Mumma, ${ }^{12}$ Kristen Nordenholz, ${ }^{13}$ Justine Pagenhardt, ${ }^{14}$ Michael Runyon, ${ }^{15}$ William Stubblefield, ${ }^{16}$ Christopher Willoughby ${ }^{17}$

To cite: Kline J, Adler D, Alanis N, et al. Study protocol for a multicentre implementation trial of monotherapy anticoagulation to expedite home treatment of patients diagnosed with venous thromboembolism in the emergency department. BMJ Open 2020;10:e038078. doi:10.1136/ bmjopen-2020-038078

- Prepublication history and additional material for this paper are available online. To view these files, please visit the journal online (http://dx.doi. org/10.1136/bmjopen-2020038078).

Received 27 February 2020 Revised 23 July 2020 Accepted 29 July 2020

D Check for updates

C Author(s) (or their employer(s)) 2020. Re-use permitted under CC BY-NC. No commercial re-use. See rights and permissions. Published by BMJ.

For numbered affiliations see end of article.

Correspondence to

Dr Jeffrey Kline; jefkline@iu.edu

\section{ABSTRACT}

Introduction In the USA, many emergency departments (EDs) have established protocols to treat patients with newly diagnosed deep vein thrombosis (DVT) as outpatients. Similar treatment of patients with pulmonary embolism $(\mathrm{PE})$ has been proposed, but no large-scale study has been published to evaluate a comprehensive, integrated protocol that employs monotherapy anticoagulation to treat patients diagnosed with DVT and PE in the ED.

Methods and analysis This protocol describes the implementation of the Monotherapy Anticoagulation To expedite Home treatment of Venous ThromboEmbolism (MATH-VTE) study at 33 hospitals in the USA. The study was designed and executed to meet the requirements for the Standards for Reporting Implementation Studies guideline. The study was funded by investigator-initiated awards from industry, with Indiana University as the sponsor. The study principal investigator and study associates travelled to each site to provide on-site training. The protocol identically screens patients with both DVT or $P E$ to determine low risk of death using either the modified Hestia criteria or physician judgement plus a negative result from the simplified PE severity index. Patients must be discharged from the ED within 24 hours of triage and treated with either apixaban or rivaroxaban. Overall effectiveness is based upon the primary efficacy and safety outcomes of recurrent VTE and bleeding requiring hospitalisation respectively. Target enrolment of 1300 patients was estimated with efficacy success defined as the upper limit of the $95 \% \mathrm{Cl}$ for the 30 -day frequency of VTE recurrence below 2.0\%. Thirty-three hospitals in 17 states were initiated in 2016-2017.

Ethics and dissemination All sites had Institutional Review Board approval. We anticipate completion of enrolment in June 2020; study data will be available after peer-reviewed publication. MATH-VTE will provide information from a large multicentre sample of US patients
Strengths and limitations of this study

- This study will be the largest implementation study of an integrated protocol to use monotherapy anticoagulation to treat venous thromboembolism conducted inside of the USA.

- This study will report the first large sample of realworld data about the efficacy and safety of home treatment with direct-acting anticoagulants for both deep vein thrombosis and pulmonary embolism initiated in the emergency department.

- A potential strength and limitation is that the integrated protocol provides the same risk stratification and emergency care treatment for patients with deep vein thrombosis and pulmonary embolism.

- A limitation is that this study is single arm with no comparator group and clinician discretion in the use of apixaban or rivaroxaban.

- Other limitations include allowance of an unstructured estimate of bleeding risk by the clinician at enrolment and outcomes only reported out to 30 days.

about the efficacy and safety of home treatment of VTE with monotherapy anticoagulation.

\section{INTRODUCTION}

This work describes the multicentre implementation of a clinical protocol to improve the transition of care of patients with venous thromboembolism (VTE, including both deep vein thrombosis, DVT, and pulmonary embolism, PE) from the emergency department (ED) to home treatment with monotherapy anticoagulation using directacting oral anticoagulants (DOACs). The 
underlying patient-oriented goal was their autonomy to manage VTE at home after immediate discharge from the ED followed by an acceptably low rate of VTE recurrence and bleeding. This project was named the Monotherapy Anticoagulation To expedite Home treatment of Venous ThromboEmbolism (MATH-VTE) protocol.

The assumptions of efficacy and safety were based on a composite of data published from 2012 to 2016 from the large Food and Drug Administration-qualifying trials for the DOACs, together with aggregated data for patients treated as outpatients with vitamin $\mathrm{K}$ antagonists (VKAs), and preliminary work with DOACs used to treat patients in ED with VTE as outpatients. For example, the 30-day incidence of major bleeding was $<1.0 \%$ in both the AMPLIFY and EINSTEIN trials, and for patients with low risk of bleeding, the rate can be expected to be about $0.5 \%$ with upper limit $95 \% \mathrm{CI}<1.00 .{ }^{1}$ Additionally, in 2015, the 90-day incidence of the composite rate for VTE recurrence and major bleeding reported in systematic reviews and meta analyses of outcomes of low-risk patients with VTE diagnosed in the ED and treated at home with VKAs was $18 / 785$ (2.2\%, 95\% CI $1.4 \%$ to $3.6 \%) .^{2}$ den Exter et al randomised 275 patients who were Hestia negative to be treated with VKAs at home; 90-day follow-up identified three patients with recurrent VTE and three with major bleeding $(6 / 275,2.2 \%) .{ }^{3}$ The LoPE study found only 1 of 200 patients with confirmed PE enroled at five hospitals suffered the composite outcome of mortality, recurrent VTE and major bleeding PE $(0.5 \%, 95 \% \mathrm{CI}=0.02 \%$ to $2.36 \%) .{ }^{4}$ (Note: Although the LoPE study was published in 2018, it was performed by an author on the present work, and the results were known in 2016). These data supported the hypothesis of an upper limit 30-day failure rate of VTE recurrence $<2.0 \%$ and bleeding $<2.0 \%$ to constitute a reasonable and prudent expectation of standard care. Evidence in 2015 (the time of study design) indicated preliminary evidence of the safety, acceptance and economic benefit of monotherapy home treatment of both DVT and PE with DOACs. ${ }^{5-7}$ However, in 2016, and still in 2020, clinicians remain(ed) wary of discharging patients with VTE in part due to a lack of a protocolised system to guarantee patient follow-up, as well as lack of experience with using DOACs, and concern about drug access and adherence. Pilot work for this project used a dedicated thrombosis clinics for follow-up in Indianapolis, ${ }^{5}$ but with more experience, the investigators found that primary care providers became more comfortable with patients with both DVT and PE being discharged directly from the ED and following up in their offices. The clinical processes for this study required three main components: selection of low-risk patients with VTE, access to a DOAC to ensure monotherapy anticoagulation (ie, no need for heparin injections) and follow-up to either a dedicated thrombosis clinic, or primary care.

\section{What does this study add in $\mathbf{2 0 2 0 ?}$}

We believe that clinicians remain wary of discharging PE from the ED. A 2019 systematic review of the topic of outpatient treatment of PE concluded 'Currently, only low-quality evidence is available from two published randomised controlled trials on outpatient versus inpatient treatment in low-risk patients with acute PE. The studies did not provide evidence of any clear difference between the interventions in overall mortality, bleeding and recurrence of $\mathrm{PE}^{\prime} .{ }^{8}$ Limited multicentre data allow an inference into the risks and benefits of outpatient treatment of DVT or PE from the emergency care setting. For DVT, available literature suggests that far fewer than onehalf of eligible patients with DVT are treated as outpatients. ${ }^{9}$ Regarding PE, Vinson et al randomised 21 sites in the Kaiser health system to either receive a targeted implementation strategy for outpatient treatment of PE $(\mathrm{n}=11$ sites $)$ or usual care $(\mathrm{n}=10)$, and found that implementation of the protocol resulted in $28 \%$ of eligible patients with PE being treated at home in the EDs with the intervention, representing a $13 \%$ increase compared with hospitals using usual care ${ }^{10}$ However, that study used VKAs as the primary modality of anticoagulation. The available literature shows a lack of real-world efficacy and safety data from an integrated DOAC-based monotherapy home treatment protocol for both DVT and PE.

\section{Theoretical framework}

We hypothesised a patient and public health benefit of implementing outpatient treatment based on VTE treatment quality measures and patient-centred outcomes. Regarding improvements in VTE treatment quality measures, propensity-matched studies suggested benefits to home treatment for DVT and PE included less bleeding and fewer VTE recurrences. ${ }^{112}$ Regarding patient-centred outcomes, patient interviews in Indianapolis, Indiana, suggested patient preference for the autonomy of monotherapy anticoagulation, and quality of life measurements that were equal for patients with DVT and PE. ${ }^{6}$ Also, several case-control reports have indicated lower overall charges for patients treated at home with DOACs, versus those treated with VKAs. ${ }^{7} 1314$ The major question was whether this protocol could be disseminated across many EDs with a wide range of geographic and demographic diversity, including community EDs, while maintaining a low 30-day rate of recurrent VTE and bleeding requiring hospitalisation, with the latter two outcomes used to determine overall effectiveness. We undertook a multifaceted approach to enhance behaviour change, including education (didactic session and publications), advertisement (placards), electronic order sets, change in culture (multidisciplinary acceptance of sending DVT and PE home), system changes (prespecified clinical follow-up) and research procedures (Institutional Review Board (IRB) approval, protocolised data collection and archiving, 30-day follow-up). ${ }^{15} 16$

\section{METHODS AND ANALYSIS}

The methods were designed to answer this research question: can ED patients with acute PE and/or DVT, 
identified as low risk by objective criteria, be treated at home with monotherapy anticoagulation with a low rate of objectively confirmed recurrent VTE requiring rehospitalisation, and low rate of bleeding requiring rehospitalisation within 30 days?

\section{Patient and public involvement}

Neither patients nor the public were directly involved in the design, recruitment nor conduct of the study.

\section{Patient selection}

Patients over 18 years could be deemed low risk by either the modified Hestia criteria or clinical judgement plus the simplified pulmonary embolism severity index (sPESI) criteria. The predicate work by the research team used the Hestia criteria for both DVT and PE with awareness that Hestia was designed and initially validated for patients with PE. ${ }^{517}$ Rationale for using the Hestia criteria for patients with DVT is based on our survey and in observation of practice; emergency physicians will typically not discharge patients with DVT who fail any of the Hestia criteria. Moreover, because many patients with DVT may have asymptomatic $\mathrm{PE}$, and in absence of pulmonary vascular imaging, clinicians cannot determine which patients with DVT have silent PE, the safest option is to screen all as if they have PE. ${ }^{18}{ }^{19}$ Also, we modified two components of the original Hestia score to be sensible in the US emergency care setting: we stated the questions in the positive (answers had to be yes), changed the requirement of ' $>24$ hours on supplemental oxygen required to maintain $\mathrm{SaO}_{2}>90 \%$ ' to 'Pulse oximetry reading $>94 \%$ ', and 'Severe pain needing intravenous pain medication required $>24$ hour' to 'More than two doses of intravenous narcotics in ED'. For the purpose of widening the inclusion criteria while maintaining safety, the steering committee recognised that the sPESI score was (and still is in 2020) well-validated to predict low-risk of death within 30 days. ${ }^{20}{ }^{21}$ However, unlike Hestia, the sPESI does not explicitly require clinicians to assess social determinants, which in the US emergency care setting, remains crucial to adherence to therapy. ${ }^{22}$ Accordingly, we added the introductory requirement to the sPESI criteria in box 1 . One publication from 2019 found the two methods equal in terms of safety. ${ }^{23}$

For patients with active cancer (defined as 'currently under the care of an oncologist'), the protocol suggested that patients be excluded if they had a probability of death $>5 \%$, as estimated by the prediction of mortality from pulmonary embolism in cancer (POMPE-C) tool. ${ }^{24}$ To assess low bleeding risk, clinicians could use their own unstructured estimate of a non-high probability of bleeding in 30 days, or the protocol suggested as an alternative defining low risk using the method of RuízGiménez et al. ${ }^{25}$

\section{Primary outcomes}

The primary efficacy outcome was VTE recurrence rate requiring hospitalisation within 30 days of enrolment,

\section{Box 1 Criteria for low risk}

The modified Hestia criteria (all must be true):

Systolic blood pressure $>100 \mathrm{~mm} \mathrm{Hg}$

No thrombolysis needed

No active bleeding

$\mathrm{SaO}_{2}>94 \%$ while breathing room air

Not already anticoagulated

No more than two doses of intravenous narcotics in the emergency department

Other medical or social reasons to admit

Creatine clearance $>30 \mathrm{~mL} / \mathrm{min}$

Not pregnant, severe liver disease or heparin-induced thrombocytopaenia OR

Physician judgement plus simplified pulmonary embolism severity index (sPESI) criteria

The physician opinion that a patients' overall social and medical situation is favourable for home treatment and the patient has a zero score on the sPESI.

All of the following must true:

Age 18-81 years

No history of cancer

No history of heart failure or chronic lung disease

Pulse $<110$ beats/min

Systolic blood pressure $>99 \mathrm{~mm} \mathrm{Hg}$

$\mathrm{O}_{2}$ saturation $>89 \%$

with success defined as an upper limit of the $95 \%$ CI for the point estimate below $2.0 \%$. The $2 \%$ upper limit of the $95 \% \mathrm{CI}$ threshold for VTE recurrence is justified based on data from AMPLIFY and pooled data from EINSTEIN DVT and PE which show a point estimate 30-day VTE recurrence rate of $1 \%-1.2 \% \cdot{ }^{2627}$ For patients deemed low risk as defined in box 1 , we predicted this rate would be considerably lower. The primary safety outcome was bleeding requiring hospitalisation within 30 days. The definition of safety success was also an upper limit of the $95 \%$ CI for the point estimate of the frequency of bleeding within 30 days below $2.0 \%$. This definition of bleeding was meant to be simple, pragmatic and patient centred, and for completeness, we will simultaneously report cases of haemorrhage classified as major and clinically relevant non-major bleeding using International Society for Thrombosis and Haemostasis (ISTH) criteria. We did not measure patient-centred outcomes such as quality of life, or qualitative data. Although not a clinical trial, this protocol was registered on clinicaltrials.gov (NCT03404635).

\section{Other outcomes}

Explicitly stated secondary objectives included the frequency of discontinuation of rivaroxaban or apixaban in the first month as reported by subject or medical record, and the total number of days of hospital stay in first 30 days. We also seek to determine the relative frequency of PE versus DVT home treatment in realworld emergency care, and to compare failure rates for the primary efficacy and safety outcomes between patients with DVT versus PE. Exploratory objectives 
included the qualitative documentation of the reasons stated by the patient for discontinuation of rivaroxaban or apixaban, alternative anticoagulation measures used and rate of discontinuation of the alternative anticoagulant. Categorical reasons for discontinuation will include: physician discretion, subject preference without physician oversight, bleeding complication, other suspected medication side effect, financial difficulties with obtaining drug, VTE recurrence on therapy, other reason. Other goals were to quantify the anatomic locations of thromboses, including the vessels obstructed by PE, frequency of arm versus leg DVT, and venous site(s) of DVT.

\section{Study design}

This is a prospective, multicentre implementation study of the effectiveness of a standard of care protocol implemented to enhance home treatment of VTE. The methods were designed to be responsive to the data elements in the Standards for Reporting Implementation guidelines checklist. ${ }^{1528}$ Study population will be selected as part of usual care as eligible for home treatment. Follow-up will be 30 days using medical records and/or telephone interview to assess for primary outcomes of bleeding or VTE recurrence. Figure 1 shows the flow diagram that was presented to each site.

\section{Site training}

This work was initiated by submission of the IIS applications in mid 2015. In April 2016 the first of 33 sites were onboarded, with the last initiated in March 2019. To facilitate a multifaceted approach, study site investigators, research staff, staff physicians and residents were provided the protocol, guidance document and other educational materials in advance of site qualification. The research team ensured that both apixaban and rivaroxaban were included as preferred drugs by state Medicaid agencies. Site investigators engaged ED stakeholders (pharmacists, social workers and case managers) in advance to streamline help with qualification from either the Johnson and Johnson Patient Assistance Foundation or the Bristol Meyers Squibb Patient Assistance Foundation to provide

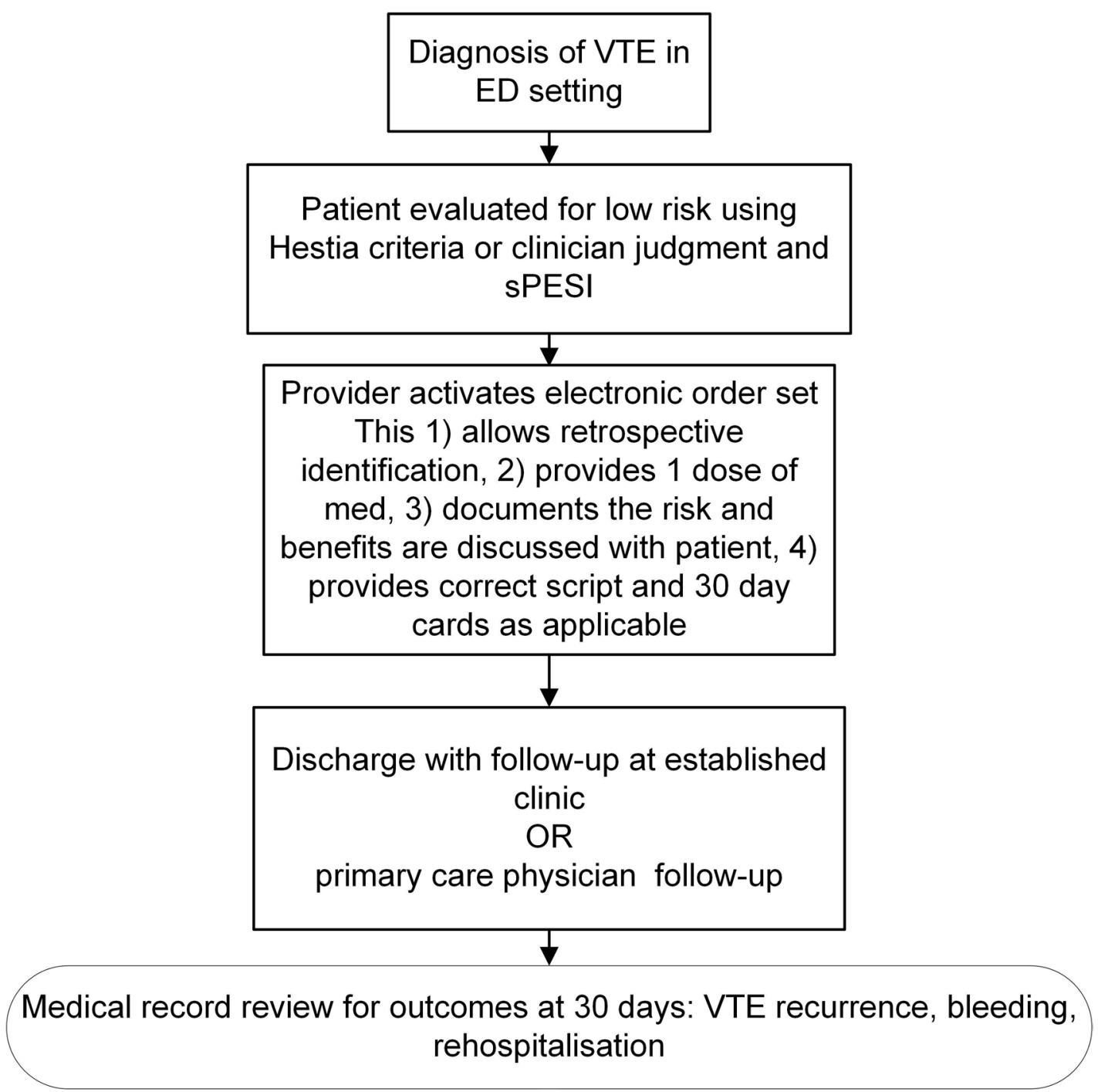

Figure 1 Overview of the protocol. ED, emergency department; sPESI, simplified pulmonary embolism severity index; VTE, venous thromboembolism. 
free drug to patients without insurance. These foundations are separate business entities from the funding agencies. All patients were eligible for 30-day free (or nominal copay) coupons offered for apixaban (https:// www.activatethecard.com/eliquis/TermsandConditions. $\mathrm{html}$ ) or rivaroxaban (https://www.janssencarepath. $\mathrm{com} /$ sites/www.janssencarepath.com/files/xarelto-textto-voucher.pdf). In some cases, local pharmaceutical representatives were contacted to ensure that ED staff had access to 30-day free drug cards to give to selected patients at discharge. Site investigators also contacted representatives of other specialties (eg, haematology, pulmonology and family medicine) to gather their input and plan for patient clinical follow-up. The study principal investigator travelled to all participating institutions to qualify the sites; deliver a Powerpoint lecture (version 1808) (online supplemental data 1) to introduce the protocol (attendees were at least the research team, and often included residents and staff physicians in a 'grand rounds' format); meet and train site principal investigators, emergency physicians and research personnel on the implementation of the protocol as part of usual clinical care; and review data collection methods for an implementation study with plans to use the data collected in this registry in future publications. The trial managers maintained and updated a guidance document that answered questions that arose as the sites began enroling (online supplemental data 2). All sites posted placards in the ED and/or physician offices, and made announcements at faculty and resident meeting to introduce protocol. To facilitate adoption postinitiation, research team members sent emails to physicians of patients who were discovered to be eligible, but who were admitted to remind them of the protocol's existence.

\section{Study population and inclusion criteria}

Patients in the ED with new or recurrent VTE deemed low-risk by modified Hestia or clinician discretion and sPESI (-) were included. Criteria for initial VTE diagnosis require a pulmonary artery filling defect interpreted as 'positive' on computerised tomographic pulmonary angiography, a ventilation-perfusion lung scan interpreted as high probability or an incompressible vein observed on venous ultrasound of an extremity (arm or leg) or jugular vein. Screening includes electronic surveillance of the 'VTE home treatment order set' which ensures low-risk criteria and then provides appropriate medication, including rivaroxaban or apixaban as an option, and discharge instructions. Enrolment occurs at the time of discharge. Patients must be discharged $<24$ hours after triage in an ED visit with diagnosis of VTE using objective criteria in the ED. Non-high risk for haemorrhage was defined by either physician implicit definition, or a score $<1.5$ using the method of Ruíz-Giménez et al (Recent major bleeding: 2 points, Creatinine levels $>1.2 \mathrm{mg} / \mathrm{dL}$ : 1.5 points, Anaemia: 1.5 points, Cancer: 1 point, Clinically overt PE: 1 point and Age $>75$ years: 1 point). ${ }^{25}$

\section{Exclusion criteria}

Patients were ineligible if the VTE diagnosis was made while taking anticoagulants with evidence of compliance (eg, physician opinion that patient is taking Eliquis, Xarelto or Pradaxa, low-molecular weight heparin injections or warfarin as prescribed for any condition). Other exclusions included sensitivity or contraindication to use of rivaroxaban or apixaban.

\section{Data source/Data collection process}

Figure 2 demonstrates the data flow process. For each patient enroled, study personnel complete an electronic case report form that is linked by a study ID (eg, CMC001) to the study site enrolment registry, maintained at the study site (REDcap) ${ }^{29}$ Placards were posted in the ED with a flow diagram similar to the one diagrammed above. Each site created a specific 'VTE home treatment' order set (in some cases, a dot phrase) which prints prescriptions or sends them electronically to a pharmacy. Online supplemental data 3 show four examples taken directly from the electronic medical record at three participating institutions. Subjects are instructed to follow-up in designated anticoagulation centres or with a primary care physician. The duration of anticoagulation is generally suggested to be a minimum of 3 months. ${ }^{30}$ Patients are identified by query of the electronic order set. Outcomes are assessed by medical record review and/ or a telephone call to each patient made after 30 days after discharge from the ED to document outcomes from the time of discharge to 30 days thereafter. Participants can be enroled after discharge, as long as they met all inclusion and no exclusion criteria.

\section{Methods of assessing outcomes}

Trial effectiveness was determined by the combination of a primary efficacy and a primary safety outcome, assessed by the composite of clinic visit results (which require a qualified healthcare provider to specifically complete a data form to assess for the primary outcomes), telephone survey and medical record review at or after 30 days, asking the subject explicitly about any change in health status, any unscheduled visit to an ED or other healthcare provider, rehospitalisations, VTE diagnoses or bleeding events. The script and procedures for the phone call, including handling disconnected numbers, and nonanswers are addressed in the guidance document (online supplemental data 2)

The definitions of recurrent VTE were based on chart review to confirm suspected recurrent PE or DVT, and the requirement of explicit radiographic or ultrasonic evidence of PE/DVT. These explicit image-based definitions of recurrent VTE, in terms of findings on imaging are in online supplemental data 4.

The definition of rehospitalisation for bleeding requires chart review demonstrating explicit written decision-making by the admitting emergency physician that a patient was admitted (requiring $>24$ hour stay) for medical or procedural care to manage objective or 


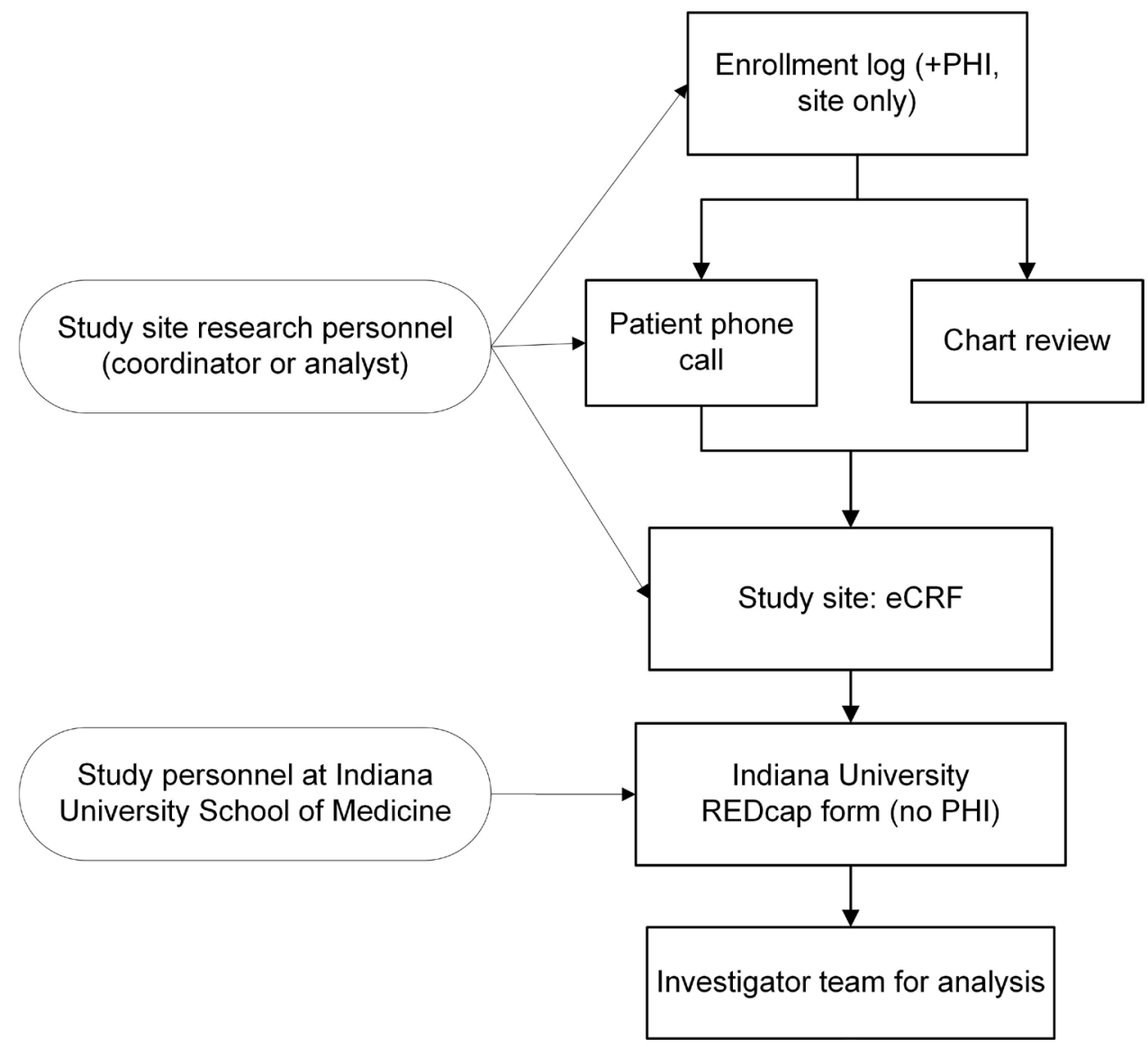

Figure 2 Diagram of data flow. PHI, protected health information.

suspected bleeding. This would include charted evidence of initially suspected but later disproven bleeding such as gastrointestinal bleeding. Bleeding events will be further characterised as major bleeding or clinically relevant non-major bleeding, using published criteria, further defined in the guidance document and using previously established criteria according to guidelines published by ISTH. $^{131}$

To assess secondary objectives, study associates use a combination of patient report and medical record documentation to determine if patients discontinued rivaroxaban or apixaban. During the follow-up telephone call, patients are asked if they discontinued rivaroxaban or apixaban and if so, what anticoagulant they are currently taking.

To assess exploratory aims, patients are asked if they are still taking rivaroxaban or apixaban, and if the answer is no, they will be asked why not and the answer is documented verbatim. This response is cross-checked by the site PI with the medical record and categorised by the site PI as 1: bleeding, 2: other side effect, 3: change in diagnosis, 4: new contraindication, 5: patient elected, 6: physician decision, not otherwise explained or 7: other

\section{Adverse events}

Adverse events are recognised by chart review or patient report. A possible adverse event is triggered by study associate recognition of a finding in the medical record or subject response suggesting a change in health status at the 30-day follow-up. The definitions of adverse events and serious adverse events followed the guidance provided by Good Clinical Practice/International Committee on Harmonization and EU Guidelines on Pharmacovigilance for Medicinal Products for Human Use.

\section{Statistical analysis methods}

The primary analysis consisted of the point estimate of the treatment failure rate (either VTE recurrence or major or clinically relevant non-major bleeding requiring hospitalisation) at 30 days with $95 \%$ CIs calculated from the exact binomial formula.

\section{Analysis plan for primary objective}

The primary efficacy aim will be calculated by determining the treatment failure rate, defined as the numerator of subjects treated in this protocol who are rehospitalised for $>24$ hours within 30 days of enrolment for either objectively diagnosed VTE or for major or clinically relevant non-major bleeding requiring hospitalisation, divided by the denominator of enroled subjects. Efficacy success was defined as the upper limit of the $95 \%$ CI of the proportion with treatment failure (hospitalisation for VTE within 30 days $)<2.0 \%$. Safety was defined as the upper limit of the 95\% CI of the proportion with bleeding requiring hospitalisation within 30 days $<2.0 \%$ 


\begin{tabular}{ll}
\hline Table 1 & Potential failure rates and associated Cls \\
\hline $\begin{array}{l}\text { Number of failures out of } \mathbf{1 3 0 0} \text { patients } \\
\text { treated }\end{array}$ & $\begin{array}{l}\text { Upper limit } \\
\mathbf{9 5 \%} \mathbf{~ C l}\end{array}$ \\
\hline 0 & 0.3 \\
5 & 0.9 \\
10 & 1.4 \\
15 & 1.9 \\
\hline
\end{tabular}

\section{Analysis plan for exploratory objectives}

Descriptive statistics with $95 \%$ CIs will be used, where appropriate, with comparison of proportions (eg, proportion of patients with VTE treated at home preimplementation versus postimplementation) using 95\% CIs for difference in proportions.

\section{Power/Ssample size}

For the primary effectiveness and safety endpoints each require an upper limit $95 \% \mathrm{CI}<2.0 \%$ for their frequency at 30 days. $^{32}$ Table 1 shows the upper limit of the $95 \%$ CI for various assumptions with a denominator (sample size) of $n=1300$. Thus, success for efficacy requires 15 or fewer VTE recurrences requiring hospitalisation and success for safety requires 15 or fewer patients with bleeding requiring hospitalisation.

\section{ETHICS AND DISSEMINATION}

All sites obtained approval from local IRBs prior to site initiation as listed below; this study did not use a single IRB.

List of IRBs that reviewed and approved the protocol

Indiana University School of medicine IRB

IU Health Methodist Hospital

IU Health West Hospital

Sidney and Lois Eskenazi Hospital

\section{Baylor Scott \& White IRB}

Baylor University Medical Center

Baylor-Garland

Baylor-Grapevine

Baylor-Irving

Baylor-Waxahachie

University of Texas Southwestern, Dallas, Texas

\section{Intermountain Healthcare IRB}

Intermountain Medical Center

Riverton Hospital

Alta View Hospital

LDS Hospital

McKay Dee Hospital

Utah Valley Hospital

American Fork Hospital

Virginia Tech Human Research Protection Program IRB: Carillion Clinic, Roanoke, Virginia

Charlotte Mecklenburg Hospital Authority IRB: Carolinas Medical Center, Charlotte, North Carolina
George Washington University School of Medicine IRB: George Washington University, Washington DC

North Texas Regional IRB: John Peter Smith Hospital, Fort Worth, Texas

MaineHealth's IRB: Maine Medical Center, Portland, Maine

Medical University of South Carolina IRB for human research: Medical University of South Carolina Charleston, South Carolina

Northwell Health Human Research Protection Program: Northwell Health, Long Island, New Y

Northwestern IRB: Northwestern Memorial Hospital, Chicago, Illinois

LSUHSC-NO's Human Subjects Research Protection Program (HRPP) and Institutional Review Board (IRB): Spirit of Charity LSU New Orleans/Baromedical, New Orleans, Louisiana

UCLA Human Research Protection Program IRB: Ronald Reagan Medical Center University of California, Los Angelos, Los Angelos, California

UC Davis IRB: University of California, Davis, Sacremento, California

Colorado Multiple Institutional Review Board (COMIRB): University of Colorado Denver, Denver, Colorado

University of Mississippi Medical Center IRB: University of Mississippi Medical Center, Jackson, Michigan

University of Rochester Medical Center Research Subjects Review Board: University of Rochester Medical Center, Rochester, New York

University of Utah Health Sciences Center IRB: University of Utah Health Sciences Center Salt Lake City, Utah

University of Virginia Institutional Review Board for Health Sciences Research (IRB-HSR): University of Virginia, Charlottesville, Virginia

WVU Office of Human Research Protections IRB: West Virginia University, Morganton, West Virginia

Patients gave either written or verbal consent to participate. The data elements of the case report form are provided in online supplemental data 5. After an embargo period extending to 1 year after publication of the first manuscript from this work to allow access for secondary manuscripts by site investigators, all data (devoid of protected health information) will be made fully available and placed on a public repository. All participating investigators signed data use agreement that specified control of data and publication plan. We anticipate study completion in June 2020.

Author affiliations

${ }^{1}$ Emergency Medicine, Indiana University School of Medicine, Indianapolis, Indiana, USA

${ }^{2}$ Emergency Medicine, University of Rochester Medical Center, Rochester, New York, USA

${ }^{3}$ Emergency Medicine, University of North Texas Health Science Center, Fort Worth, Texas, USA

${ }^{4}$ Emergency Medicine, Intermountain Health Care Inc, Salt Lake City, Utah, USA ${ }^{5}$ Emergency Medicine, University of Texas Southwestern Medical School, Dallas, Texas, USA 
${ }^{6}$ Emergency Medicine, John Peter Smith Hospital, Fort Worth, Texas, USA ${ }^{7}$ Emergency Medicine, Baylor University Medical Center at Dallas, Dallas, Texas, USA

${ }^{8}$ Emergency Medicine, University of Mississippi Medical Center, Jackson, Mississippi, USA

${ }^{9}$ Emergency Medicine, Maine Medical Center, Portland, Maine, USA

${ }^{10}$ Emergency Medicine, University of Utah, Salt Lake City, Utah, USA

${ }^{11}$ Emergency Medicine, Medical University of South Carolina, Charleston, South Carolina, USA

${ }^{12}$ Emergency Medicine, University of California Davis, Davis, California, USA

${ }^{13}$ Emergency Medicine, University of Colorado Denver, Denver, Colorado, USA

${ }^{14}$ Emergency Medicine, West Virginia University - Health Sciences Campus,

Morgantown, West Virginia, USA

${ }^{15}$ Emergency Medicine, Atrium Health, Charlotte, North Carolina, USA

${ }^{16}$ Emergency Medicine, Vanderbilt University Medical Center, Nashville, Tennessee, USA

${ }^{17}$ Internal Medicine, Louisiana State University, New Orleans, Louisiana, USA

\section{Twitter David MacKenzie @mackendc}

Acknowledgements The authors thank the participating institutions in MATH-VTE, Indianapolis area hospitals, IU Health Methodist Hospital, IU Health West Hospital, Sidney and Lois Eskenazi Hospital, Dallas area hospitals, Baylor University Medical Center, Baylor-Garland, Baylor-Grapevine, Baylor-Irving, Baylor-Waxahachie, University of Texas Southwestern, Dallas, TX, Intermountain Healthcare, Salt Lake City, UT, Intermountain Medical Center, Riverton Hospital, Alta View Hospital, LDS Hospital, McKay Dee Hospital, Utah Valley Hospital, American Fork Hospital, Carillion Clinic, Roanoke, VA, Carolinas Medical Center, Charlotte, NC, George Washington University, Washington DC, John Peter Smith Hospital, Fort Worth, TX, Maine Medical Center, Portland, ME, Medical University of South Carolina, Charleston, SC, Northwell Health, Long Island, NY, Northwestern Memorial Hospital, Chicago, IL, Spirit of Charity LSU New Orleans/Baromedical, New Orleans, LA, University of California, Los Angelos, Los Angelos, CA, University of California, Davis, Sacremento, $\mathrm{CA}$, University of Colorado Denver, Denver, C0, University of Mississippi Medical Center, Jackson, MI, University of Rochester Medical Center, Rochester, NY, University of Utah Health, Sciences Center, Salt Lake City, UT, University of Virginia, Charlottesville, VA, West Virginia University, Morganton, WV.

Contributors JK conceived and designed the work, performed data acquisition, analysis and interpretation of data and drafted, revised and gave final approval of the work. DA participated in data acquisition, analysis and interpretation of data and assisted in drafting and revision that was critically important to the intellectual content and gave final approval of the work. NA participated in data acquisition, analysis and interpretation of data and assisted in drafting and revision that was critically important to the intellectual content and gave final approval of the work. JB participated in data acquisition, analysis and interpretation of data and assisted in drafting and revision that was critically important to the intellectual content and gave final approval of the work. DM participated in data acquisition, analysis and interpretation of data and assisted in drafting and revision that was critically important to the intellectual content and gave final approval of the work. JP participated in data acquisition, analysis and interpretation of data and assisted in drafting, revision that was critically important to the intellectual content and gave final approval of the work. DBD participated in data acquisition, analysis and interpretation of data and assisted in drafting and revision that was critically important to the intellectual content and gave final approval of the work. JG participated in data acquisition, analysis and interpretation of data and assisted in drafting, revision that was critically important to the intellectual content and gave final approval of the work. AEJ participated in data acquisition, analysis and interpretation of data and assisted in drafting, revision that was critically important to the intellectual content and gave final approval of the work. DM participated in data acquisition, analysis and interpretation of data and assisted in drafting, revision that was critically important to the intellectual content and gave final approval of the work. TM participated in data acquisition, analysis and interpretation of data and assisted in drafting, revision that was critically important to the intellectual content and gave final approval of the work. AM participated in data acquisition, analysis and interpretation of data and assisted in drafting, revision that was critically important to the intellectual content and gave final approval of the work. BM participated in data acquisition, analysis and interpretation of data and assisted in drafting, revision that was critically important to the intellectual content and gave final approval of the work. KN participated in data acquisition, analysis and interpretation of data and assisted in drafting, revision that was critically important to the intellectual content and gave final approval of the work.
JP participated in data acquisition, analysis and interpretation of data and assisted in drafting, revision that was critically important to the intellectual content and gave final approval of the work. MR participated in data acquisition, analysis and interpretation of data and assisted in drafting, revision that was critically important to the intellectual content and gave final approval of the work. WS participated in data acquisition, analysis and interpretation of data and assisted in drafting, revision that was critically important to the intellectual content and gave final approval of the work. CW participated in data acquisition, analysis and interpretation of data and assisted in drafting, revision that was critically important to the intellectual content and gave final approval of the work.

Funding This work was funded by two separate investigator-initiated studies (IIS) to pharmaceutical companies, one from Janssen Scientific Affairs, LLC and the second from Pfizer-BMS with all funding to Indiana University School of Medicine. None of the investigators received money for the work, and none work as consultants or serve on speaker's bureaus for the companies. Both IIS applications were written and submitted by an author (JK) in 2015. The protocols and data collection forms were identical in the two IIS applications and at the time of award, the protocols appended to the contracts were identical with one exception: the Janssen award allowed either apixaban or rivaroxaban, but the Pfizer-BMS specified that only patients treated with apixaban could be included. Additionally, the two funding sources required different methods and locations to report serious adverse events for apixaban versus rivaroxaban. The funding sources otherwise had no role in study design, inclusion criteria, outcomes, methodology of implementation, the choice of sites, protocol execution or data collection. Each contract specified that the sponsor of record was Indiana University. Sites were paid by Indiana University on a capitated basis based on patient enrolment milestones.

Competing interests None declared.

Patient consent for publication Not required.

Provenance and peer review Not commissioned; externally peer reviewed.

Open access This is an open access article distributed in accordance with the Creative Commons Attribution Non Commercial (CC BY-NC 4.0) license, which permits others to distribute, remix, adapt, build upon this work non-commercially, and license their derivative works on different terms, provided the original work is properly cited, appropriate credit is given, any changes made indicated, and the use is non-commercial. See: http://creativecommons.org/licenses/by-nc/4.0/.

ORCID iDs

Jeffrey Kline http://orcid.org/0000-0001-7190-3109

Naomi Alanis http://orcid.org/0000-0003-2200-315X

\section{REFERENCES}

1 Kline JA, Jimenez D, Courtney DM, et al. Comparison of four bleeding risk scores to identify Rivaroxaban-treated patients with venous thromboembolism at low risk for major bleeding. Acad Emerg Med 2016;23:144-50.

2 Yoo HH, Queluz TH, DR E. Outpatient versus inpatient treatment for acute pulmonary embolism. CochraneDatabaseSystRev 2014:11:CD010019.

3 den Exter PL, Zondag W, Klok FA, et al. Efficacy and safety of outpatient treatment based on the Hestia clinical decision rule with or without $\mathrm{N}$-terminal pro-brain natriuretic peptide testing in patients with acute pulmonary embolism. A randomized clinical trial. Am J Respir Crit Care Med 2016;194:998-1006.

4 Bledsoe JR, Woller SC, Stevens SM, et al. Management of low-risk pulmonary embolism patients without hospitalization: the lowrisk pulmonary embolism prospective management study. Chest 2018;154:249-56.

5 Beam DM, Kahler ZP, Kline JA. Immediate discharge and home treatment with rivaroxaban of low-risk venous thromboembolism diagnosed in two U.S. emergency departments: a one-year Preplanned analysis. Acad Emerg Med 2015;22:788-95.

6 Kline JA, Kahler ZP, Beam DM. Outpatient treatment of low-risk venous thromboembolism with monotherapy oral anticoagulation: patient quality of life outcomes and clinician acceptance. Patient Prefer Adherence 2016;10:561-9.

7 Kahler ZP, Beam DM, Kline JA. Cost of treating venous thromboembolism with heparin and warfarin versus home treatment with rivaroxaban. Acad Emerg Med 2015;22:796-802.

8 Yoo HH, Nunes-Nogueira VS, Fortes Villas Boas PJ, et al. Outpatient versus inpatient treatment for acute pulmonary embolism. Cochrane Database Syst Rev 2019;3:Cd010019. 
9 Douce D, McClure LA, Lutsey P, et al. Outpatient treatment of deep vein thrombosis in the United States: the reasons for geographic and racial differences in stroke study. J Hosp Med 2017;12:826-30.

10 Vinson DR, Mark DG, Chettipally UK, et al. Increasing safe outpatient management of emergency department patients with pulmonary embolism: a controlled pragmatic trial. Ann Intern Med 2018;169:855-65.

11 Lozano F, Trujillo-Santos J, Barrón M, et al. Home versus in-hospital treatment of outpatients with acute deep venous thrombosis of the lower limbs. J Vasc Surg 2014;59:1362-7.

12 Roy P-M, Corsi DJ, Carrier M, et al. Net clinical benefit of hospitalization versus outpatient management of patients with acute pulmonary embolism. J Thromb Haemost 2017;15:685-94.

13 Margolis JM, Deitelzweig S, Kline J, et al. Shorter Hospital stays and lower costs for rivaroxaban compared with warfarin for venous thrombosis admissions. J Am Heart Assoc 2016;5:003788.

14 Margolis JM, Deitelzweig S, Kline J, et al. Pulmonary embolism inpatients treated with rivaroxaban had shorter Hospital stays and lower costs compared with warfarin. Clin Ther 2016;38:2496-503.

15 Neta G, Glasgow RE, Carpenter CR, et al. A framework for enhancing the value of research for dissemination and implementation. Am J Public Health 2015;105:49-57.

16 Grimshaw JM, Shirran L, Thomas R, et al. Changing provider behavior: an overview of systematic reviews of interventions. Med Care 2001;39:li2-45.

17 Zondag W, den Exter PL, Crobach MJT, et al. Comparison of two methods for selection of out of hospital treatment in patients with acute pulmonary embolism. Thromb Haemost 2013;109:47-52.

18 Tzoran I, Saharov G, Brenner B, et al. Silent pulmonary embolism in patients with proximal deep vein thrombosis in the lower limbs. $J$ Thromb Haemost 2012:10:564-71.

19 Stein PD, Matta F, Musani MH, et al. Silent pulmonary embolism in patients with deep venous thrombosis: a systematic review. Am J Med 2010;123:426-31.

20 Konstantinides SV, Torbicki A, Agnelli G, et al. Esc guidelines on the diagnosis and management of acute pulmonary embolism: the task force for the diagnosis and management of acute pulmonary embolism of the European Society of cardiology (ESC) endorsed by the European respiratory Society (ERS). EurHeart J 2014:ehu283.

21 Konstantinides SV, Meyer G, Becattini C, et al. Esc guidelines for the diagnosis and management of acute pulmonary embolism developed in collaboration with the European respiratory Society (ERS): the task force for the diagnosis and management of acute pulmonary embolism of the European Society of cardiology (ESC). Eur Heart $J$ 2019

22 Davis DP, Jandrisevits MD, lles S, et al. Demographic, socioeconomic, and psychological factors related to medication nonadherence among emergency department patients. J Emerg Med 2012;43:773-85.

23 Quezada CA, Bikdeli B, Villén T, et al. Accuracy and interobserver reliability of the simplified pulmonary embolism severity index versus the Hestia criteria for patients with pulmonary embolism. Acad Emerg Med 2019;26:394-401.

24 Kline JA, Roy P-M, Than MP, et al. Derivation and validation of a multivariate model to predict mortality from pulmonary embolism with cancer: the POMPE-C tool. Thromb Res 2012;129:e194-9.

25 Ruíz-Giménez N, Suárez C, González R, et al. Predictive variables for major bleeding events in patients presenting with documented acute venous thromboembolism. findings from the RIETE registry. Thromb Haemost 2008;100:26-31.

26 Agnelli G, Buller HR, Cohen A, et al. Oral apixaban for the treatment of acute venous thromboembolism. N Engl J Med 2013;369:799-808.

27 Buller HR, Prins MH, Lensin AW, et al. Oral rivaroxaban for the treatment of symptomatic pulmonary embolism. NEnglJ Med 2012;366:1287-97.

28 Pinnock H, Barwick M, Carpenter CR, et al. Standards for reporting implementation studies (STARI): explanation and elaboration document. BMJ Open 2017;7:e013318.

29 Harris PA, Taylor R, Thielke R, et al. Research electronic data capture (REDCap)--a metadata-driven methodology and workflow process for providing translational research informatics support. J Biomed Inform 2009;42:377-81.

30 Kearon C, Akl EA, Ornelas J, et al. Antithrombotic therapy for VTe disease: chest guideline and expert panel report. Chest 2016;149:315--52.

31 Kaatz S, Ahmad D, Spyropoulos AC, et al. Definition of clinically relevant non-major bleeding in studies of anticoagulants in atrial fibrillation and venous thromboembolic disease in non-surgical patients: communication from the SSC of the ISTH. J Thromb Haemost 2015;13:2119-26.

32 Arkin CF, Wachtel MS. How many patients are necessary to assess test performance? JAMA 1990;263:275-80. 